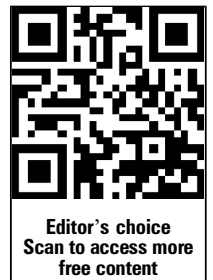

1 Division of Global Public Health, Department of Medicine, University of California, San Diego, La Jolla, California, USA

${ }^{2}$ Division of Adolescent Medicine, Department of Pediatrics, School of Medicine, Children's Hospital of Pittsburgh, University of Pittsburgh, Pittsburgh, Pennsylvania, USA

${ }^{3}$ Department of Population, Family, and Reproductive Health, Johns Hopkins Bloomberg School of Public Health, Baltimore, Maryland, USA

\section{Correspondence to} Dr Elizabeth Reed

Division of Global Public Health, Department of Medicine, University of California, San Diego, 9500 Gilman Drive, La Jolla, CA 92093, USA:

lizreed@ucsd.edu

Received 15 January 2013 Revised 12 September 2013 Accepted 23 January 2014 Published Online First 27 February 2014

\title{
Teen dating violence perpetration and relation to STI and sexual risk behaviours among adolescent males
}

\author{
Elizabeth Reed, ${ }_{1}^{1}$ Elizabeth Miller, ${ }^{2}$ Anita Raj, ${ }_{1}^{1}$ Michele R Decker, ${ }^{3}$ Jay G Silverman ${ }^{1}$
}

\begin{abstract}
Objectives To investigate teen dating violence (TDV) perpetration (physical, sexual or psychological violence) and association with STI and related sexual risk behaviours among urban male adolescents.

Methods Adolescent male survey participants $(N=134)$ were aged 14-20 years, recruited from urban health centres. Using crude and adjusted logistic regression, TDV perpetration was examined in relation to selfreported: STI, having sex with another person when they were only supposed to have sex with their main partner, and consistent condom use.
\end{abstract}

Results Over one-third of males (45\%) reported any TDV; $42 \%$ reported sexual violence perpetration, 13\% reported perpetrating physical violence against a dating/ sexual partner and $11 \%$ reported psychological violence, including threats of physical or sexual violence. Approximately $15 \%$ of males reported having ever had an STI, one quarter reported having sex with another person when they were only supposed to have sex with their main partner and 36\% reported consistent condom use (past 3 months). In adjusted logistic regression models, TDV perpetration was significantly associated with self-reports of an STI (OR=3.3; $95 \% \mathrm{Cl} 1.2$ to 9.2) and having sex with another person when they were supposed to be only having sex with their main partner (OR=4.8; 95\% Cl 2.0 to 11.4). There was no significant association between TDV perpetration and consistent condom use.

Conclusions Current study findings are the first within the literature on adolescents to suggest that greater STI and sexual risk behaviours among male adolescents perpetrating TDV may be one mechanism explaining increased STI among female adolescents reporting TDV victimisation.

\section{INTRODUCTION}

Teen dating violence (TDV) is a critical threat to public health with well-documented physical, sexual and psychological health consequences among female adolescents. ${ }^{1-3}$ Multiple studies have established the relation between girls' experiences of male perpetrated TDV and increased risk for HIV/STI. ${ }^{1} 2$

Understanding the mechanisms underlying this finding is especially critical among adolescents, given that youth between the ages of 15 and 24 acquire nearly half of all new STIs, despite representing only one quarter of the sexually active population. ${ }^{4}$ It has been well documented that girls' reduced sexual negotiating power (eg, for condom use, other sexual decision making) in abusive relationships is one contributing factor. ${ }^{2}$
However, recent research among adults has indicated that males reporting partner violence perpetration are significantly more likely than non-perpetrators to test positive for HIV/STI and to report non-condom use. ${ }^{5} 6$ Thus, greater reports of STI and sexual risk behaviours among adult males reporting perpetration also appear to explain greater HIV/STI among female adults reporting male-perpetrated victimisation. To date, no published work has documented this finding among adolescents. The current study aims to investigate TDV perpetration and relation to STI and related sexual risk behaviours among an urban clinic sample of adolescent males.

\section{METHODS}

\section{Sample and data collection}

The current cross-sectional study was conducted via an anonymous survey of English and Spanish-speaking males ages 14-20 years recruited from adolescent health clinics (providing general health services) in urban neighbourhoods of Greater Boston $(n=320)$ as part of a larger study among male and female adolescents to understand the mechanisms contributing to the association between TDV victimisation and STI as well as pregnancy among girls and young women. Data were collected in five clinics providing confidential services to adolescents in a major urban metropolitan area; two clinics were located in schools, one was located within a community health centre and two were based in other community settings. Upon arrival to the clinic, adolescent males seeking health services of any type were screened for age eligibility by trained research staff. A significant proportion of males reported coming in to access condoms $(30 \%)$, 5\% reported coming in for STI testing and $14 \%$ reported coming in for an annual exam; the remaining reported coming in as a result of seeking care or follow-up for other health issues. Eligible males indicating an interest in participating were escorted to a private area of the clinic for consent procedures and survey data collection. The participation rate for males who were approached to take the survey was $68 \%$. A subsample of 134 males who reported ever having sex with a female partner was included in the current study analyses.

Data were collected via Audio Computer Assisted Self Interview (ACASI), allowing participants to complete surveys on a computer with questions read aloud to them over headphones. Surveys asked participants details of their family, friends, employment and school, as well as information on history of dating, TDV perpetration and sexual risk
Raj A, et al. Sex Transm Infect 2014;90:322-324 
behaviours. Following survey completion, all participants were screened for psychological distress and provided with a list of local resources (eg, violence and other support services). All study procedures were approved by research ethics committees at the Partners Health Care System, Cambridge Health Alliance and the Harvard School of Public Health.

\section{Measures}

Single items assessed demographic characteristics, including age (years), race/ethnicity, education level, nativity (US born), living situation (eg, living with family, friends or partners), as well as age characteristics of their last female sex partner. Age of last sex partner (in years) was also included to understand whether boys' risk for STI has implications for STI risk among girls who are within the same age group.

TDV perpetration was assessed by whether boys' reported perpetrating one of the following: (1) sexual violence or (2) physical violence, or (3) psychological violence, including threats of physical or sexual violence. Items asked boys to report these behaviours regarding girls they "have gone out with, hooked up with, or had sex with". Items were informed by qualitative interviews previously conducted with a similar sample of adolescent boys reporting TDV perpetration ${ }^{7}$ and developed by our team specifically for use within this urban population. Psychological violence measures and threats of violence items were highly correlated; however, to maintain a conservative approach in defining TDV perpetration, only boys reporting both were categorised as perpetrating TDV. Sexual violence was measured by four items asking boys if they have ever had sex with a girl as a result of "playing mind games, using threats, using force, or she was too high/drunk to stop you". These four items were asked for different types of sexual activity, including vaginal sex, oral sex anal sex or other sexual activity. If boys reported any of these, they were categorised as having perpetrated sexual violence. Physical violence was measured by four items assessing boys' reports of (1) hitting, kicking, slapping, punching, shoving or throwing something at a girl; (2) slamming a girl against a wall; (3) choking a girl; or (4) using a gun on a girl. Boys who reported one of these items were categorised as having perpetrated physical violence. Psychological violence was measured by boys' reports of any one of the following: excessive jealousy (telling a girl that "they better not call or talk to a friend of theirs or call or talk to other boys"), destroying the property of a partner or swore at or insulted a partner (called them stupid, ugly or fat). Threats of violence was measured by two items asking boys whether they had ever (1) threatened to hurt a girl physically or (2) threatened to rape them.

Boys' reports of STI were measured by asking participants if they have ever been told by a doctor they had an STI (including Chlamydia, gonorrhoea, syphilis, herpes, genital warts, hepatitis B or crabs). An item also asked whether boys reported having "sex with someone else when you were supposed to only be having sex with the person you were dating or going out with" in the past 12 months. Consistent condom use was measured by asking males how often they used condoms during sex in the last 3 months, with five-point Likert scale response categories ranging from 'always' to 'never '. Males who reported to 'always' use condoms were categorised as having consistent condom use in the past 3 months.

\section{Data analysis}

Crude and adjusted logistic regression models were used to examine TDV perpetration in relation to (a) STI history, (b) having sex with another person when they were only supposed to have sex with their main partner and (c) condom non-use during last sex. All demographic variables (age, race/ethnicity, education level, nativity and living situation) were examined in relation to these outcomes $(\mathrm{p}<0.05)$; those that were statistically significant in relation to any of these outcomes were included in adjusted regression analyses. All analyses were conducted using SAS V.9.1 (SAS Institute Inc, Cary, North Carolina, USA).

\section{RESULTS}

\section{Sample characteristics}

The mean age of the sample was 17 ( $\mathrm{SD}=1.8$ years). In total, $54 \%$ of the sample identified as black or African-American, 9\% as white and 3\% reported Asian race/ethnicity; 46\% identified as Hispanic or Latino. The majority $(80 \%)$ of boys were born in the USA, and most (84\%) reported living with their parents or other family. In terms of the age of the girls in which boys had sexual and/or dating relationships with (to understand whether boys' STI risk has implications for STI risk among girls within the same age group), the mean age of the last sex partner was reported to be 17 years of age $(S D=3.5)$, and $87 \%$ of the sample reported that their last sex partner was younger than 20 years of age. Over one-third of males (45\%) reported any TDV; 42\% reported sexual violence perpetration, 13\% reported perpetrating physical violence against a dating/sexual partner and $11 \%$ reported psychological violence, including threats of physical or sexual violence. Approximately $15 \%$ of males reported having ever had an STI, one quarter reported having sex with another person when they were only supposed to have sex with their main partner and 36\% reported consistent condom use (past 3 months) (table 1).

\section{Relation of TDV perpetration to STI and related sexual risk variables}

In adjusted logistic regression models, TDV perpetration was significantly associated with a history of STI $(\mathrm{OR}=3.3 ; 95 \% \mathrm{CI}$ 1.2 to 9.2 ) and having sex with another person when they were only supposed to have sex with their main partner $(\mathrm{OR}=4.8$; 95\% CI 2.0 to 11.4 ). Males who reported TDV perpetration were less likely to report consistent condom use (32\%) compared with those not reporting TDV (39\%); however, this was only a trend and not statistically significant $(\mathrm{p}=0.3)($ table 1$)$.

\section{DISCUSSION}

The current study provides preliminary evidence that greater reports of STI and sexual risk behaviours among male adolescents perpetrating TDV may be one mechanism explaining high rates of STI among female adolescents reporting TDV victimisation. This finding is the first within the literature on adolescents; however, it is consistent with the adult literature on intimate partner violence perpetration and STI risk. ${ }^{5} 6$ The high proportion reporting TDV perpetration (45\%), particularly sexual violence perpetration $(42 \%)$, has been cited in our previous work ${ }^{8}$ and is likely a result of improved reporting due to the use of measurement items within the survey that were developed specifically for use with this urban population of males (ie, these items may have been more sensitive to capture TDV perpetration within this population of urban males).

These findings should be interpreted in light of several limitations. Our data consist of self-reported responses, and thus some items may be under-reported; however, we were able to document a high proportion of TDV perpetration. Additionally, the use of ACASI is recommended as the best method to reduce 
Table 1 Teen dating violence (TDV) perpetration and relation to STI and other sexual risk behaviours: crude and adjusted logistic regression findings

\begin{tabular}{|c|c|c|c|c|c|}
\hline Variable & Total $(n=134) \%(n)$ & TDV perpetration $(n=60) \%(n)$ & No TDV perpetration $(n=74) \%(n)$ & Crude OR $(95 \% \mathrm{Cl})$ & Adjusted OR $(95 \% \mathrm{Cl})^{*}$ \\
\hline \multicolumn{6}{|c|}{ History of STI } \\
\hline Yes & $14.9(20)$ & $23.3(14)$ & $8.1(6)$ & $3.5(1.2$ to 9.6$)$ & $3.3(1.2$ to 9.2$)$ \\
\hline No & $85.1(114)$ & $76.6(46)$ & $91.9(68)$ & 1.0 Referent & 1.0 Referent \\
\hline \multicolumn{6}{|c|}{ Having sex with another person outside of their main relationship (when not supposed to) } \\
\hline Yes & $25.4(34)$ & $41.7(25)$ & $12.2(9)$ & $5.2(2.2$ to 12.3$)$ & $4.8(2.0$ to 11.4$)$ \\
\hline No & $74.6(100)$ & $58.3(35)$ & $87.8(65)$ & 1.0 Referent & 1.0 Referent \\
\hline \multicolumn{6}{|c|}{ Consistent condom use, past 3 months } \\
\hline Yes & $35.8(48)$ & $31.7(19)$ & $39.2(29)$ & $0.7(0.3$ to 1.4$)$ & $0.8(0.4$ to 1.6$)$ \\
\hline No & $64.2(86)$ & $68.3(41)$ & $60.8(45)$ & 1.0 Referent & 1.0 Referent \\
\hline
\end{tabular}

${ }^{*}$ Adjusted for living situation (whether or not boys lived with family); no other demographic variables were significantly associated with outcome variables at $\mathrm{p}<0.05$ in this fairly homogenous sample.

under-reporting of TDV and other sensitive topics. ${ }^{9}$ Clinics for this study were based in an urban metropolitan area (primarily serving clients from low-income, urban communities surrounding these health centres); thus, findings do not necessarily generalise to experiences of other groups of adolescent male clinic users. Of note, findings did not differ significantly across clinics, suggesting that the results are likely to reflect TDV prevalence among male users of teen clinics in similar urban metropolitan areas with similar demographic characteristics. Furthermore, the response rate for the clinic survey was relatively high (68\%). In summary, findings are most generalisable to similar urban clinic settings and where TDV is occurring in high proportions. To confirm and further investigate these findings related to TDV perpetration, longitudinal studies are needed, including larger and diverse samples (eg, in terms of age, clinical and community samples, etc.) and biological indicators of STI.

These limitations notwithstanding, findings from the current study are consistent with ongoing work indicating the need for TDV prevention programmes tailored for young males, ${ }^{10-12}$ and build on this work by highlighting the need for such programming to address sexual risk behaviours for STI/HIV. Findings also call for increased awareness within clinical and programmatic settings that emphasise the heightened risk for STI among females experiencing TDV. Such prevention efforts are critical for this age group, which is highly impacted by TDV and STI.

\section{Key Messages}

- Multiple studies have established the relation between experiences of teen dating violence (TDV) and increased risk for STI among female adolescents.

- Greater STI and sexual risk behaviors among males perpetrating TDV is likely one mechanism explaining high STI rates among female adolescents reporting TDV victimization.

- TDV prevention and awareness programs likely need to also address sexual risk for STI.

\section{Handling editor Jackie A Cassell}

Contributors ER created the concept of the paper and took the lead in all analysis and writing. EM worked on developing the manuscript concept and the writing of the paper. AR and MRD worked on writing the paper. JGS worked on developing the manuscript's concept and writing of the paper. All authors also worked on data collection and coordination.

Funding The research described was supported by grants to JGS from the National Center for Injury Prevention and Control/CDC (U36/CCU300430-23) and to EM from the W.T. Grant Foundation Scholars Program.

\section{Competing interests None.}

\section{Patient consent Obtained.}

Ethics approval IRB committees at Partners Health Care System, Cambridge Health Alliance and the Harvard School of Public Health.

Provenance and peer review Not commissioned; externally peer reviewed.

\section{REFERENCES}

1 Silverman JG, Raj A, Mucci LA, et al. Dating violence against adolescent girls and associated substance use, unhealthy weight control, sexual risk behavior, pregnancy, and suicidality. JAMA 2001;286:572-9.

2 Silverman JG, McCauley HL, Decker MR, et al. Coercive forms of sexual risk and associated violence enacted by male partners of female adolescents. Perspect Sex Reprod Health 2011;43:60-5

3 Catalano S. Intimate partner violence in the United States. U.S. Department of Justice, Bureau of Justice Statistics, 2007. http://www.ojp.usdoj.gov/bjs/intimate/ipv. htm

4 Weinstock H, Berman S, Cates W Jr. Sexually transmitted diseases among American youth: incidence and prevalence estimates, 2000. Perspect Sex Reprod Health 2004;36:6-10.

5 Raj $A$, Reed $E$, Welles $S L$, et al. Intimate partner violence perpetration, risky sexual behavior and STI/HIV diagnosis among heterosexual African American men. Am J Mens Health 2008;2:291-5.

6 Decker MR, Seage GR III, Hemenway D, et al. Intimate partner violence functions as both a risk marker and risk factor for women's HIV infection: findings from Indian husband-wife dyads. J Acquir Immune Defic Syndr 2009;51:593-600.

7 Reed E, Silverman JG, Raj A, et al. Social and environmental contexts of adolescent and young adult male perpetrators of intimate partner violence: a qualitative study. Am J Men's Health 2008;2:260-71.

8 Reed E, Silverman JG, Raj A, et al. Male perpetration of teen dating violence: associations with neighborhood violence involvement, gender attitudes, \& perceived peer and neighborhood norms. J Urban Health 2011;88:226-39.

9 Abbey A. Lessons learned and unanswered questions about sexual assault perpetration. J Interpers Violence 2005;20:39-42.

10 Barker G, Ricardo C, Nascimento M. Engaging men and boys in changing gender-based inequity in health: Evidence from programme interventions. Geneva, Switzerland: World Health Organization, 2007. International Center for Research on Women, Instituto Promundo. Engaging Men and Boys to Achieve Gender Equality: How Can We Build on What We Have Learned? 2007. United Nations Population Fund (UNFPA), Promundo. Engaging Men and Boys in Gender Equality and Health. 2010.

11 Miller E, Tancredi D, McCauley H, et al. 'Coaching boys into men': a cluster-randomized controlled trial of a dating violence prevention program. J Adolesc Health 2012;51:431-8.

12 Miller $\mathrm{E}$, Tancredi D, McCauley $\mathrm{H}$, et al. Twelve month follow up of a cluster-randomized controlled trial of a coach-delivered dating violence prevention program. Am J Prev Med 2013;45:108-12. 